\title{
The gain and loss of chromosomal integron systems in the Treponema species
}

\author{
Yu-Wei Wu' ${ }^{1}$, Thomas G Doak ${ }^{2}$ and Yuzhen Ye ${ }^{1 *}$
}

\begin{abstract}
Background: Integron systems are now recognized as important agents of bacterial evolution and are prevalent in most environments. One of the human pathogens known to harbor chromosomal integrons, the Treponema spirochetes are the only clade among spirochete species found to carry integrons. With the recent release of many new Treponema genomes, we were able to study the distribution of chromosomal integrons in this genus.

Results: We find that the Treponema spirochetes implicated in human periodontal diseases and those isolated from cow and swine intestines contain chromosomal integrons, but not the Treponema species isolated from termite guts. By examining the species tree of selected spirochetes (based on 31 phylogenetic marker genes) and the phylogenetic tree of predicted integron integrases, and assisted by our analysis of predicted integron recombination sites, we found that all integron systems identified in Treponema spirochetes are likely to have evolved from a common ancestor - a horizontal gain into the clade. Subsequent to this event, the integron system was lost in the branch leading to the speciation of T. pallidum and T. phagedenis (the Treponema sps. implicated in sexually transmitted diseases). We also find that the lengths of the integron attC sites shortened through Treponema speciation, and that the integron gene cassettes of $T$. denticola are highly strain specific.

Conclusions: This is the first comprehensive study to characterize the chromosomal integron systems in Treponema species. By characterizing integron distribution and cassette contents in the Treponema sps., we link the integrons to the speciation of the various species, especially to the pathogens T. pallidum and T. phagedenis.
\end{abstract}

Keywords: Chromosomal integron, Treponema species, Integron integrase, attC site

\section{Background}

As important agents of bacterial evolution, integrons are genetic elements that aggregate mobile gene cassettes via site-specific recombination. The functional platform of integron systems consists of a site-specific tyrosine recombinase (intl), its primary recombination site (attl), and a transcriptional promoter for the cassettes [1]. Integrons are capable of acquiring, rearranging, and expressing genes contained in gene cassettes sampled from a nearinfinite environmental bank of cassettes [2]. Each cassette carries one or a small number of genes (some cassettes lack open reading frames) linked to a recombination site termed attC. The genes in cassettes are highly diverse and mostly of unknown function [1], and they are usually promoterless and hence rely on the integron's promoter

\footnotetext{
* Correspondence: yye@indiana.edu

${ }^{1}$ School of Informatics and Computing, Indiana University, Bloomington, IN 47408, USA

Full list of author information is available at the end of the article
}

for transcription. Accordingly, insertions of gene cassettes in the cassette array are highly orientation specific such that integron genes are transcribed from the integron promoter. Studies have revealed that gene cassette composition is extremely dynamic within and between environments [2-5] and even between closely related strains as observed in the Vibrio cholerae species [6].

There are two primary types of integrons: mobile integrons and chromosomal integrons [1]. Mobile integrons (including integrons belonging to classes 1, 2 and 3, as defined by their respective intI genes) are found commonly on plasmids and are characterized by frequent lateral gene transfers (LGT); most other classes of integrons are nonmobile and so confined to chromosomes and specific bacterial lineages, and are therefore called chromosomal integrons [7]. First discovered in the 1980s [8], mobile integrons contain mostly antibiotic resistance genes [9] and their ability to acquire a number of resistance cassettes leads to most clinical

\section{Biomed Central}


multidrug resistance. By contrast, chromosomal integrons were first found in Vibrio cholerae in the 1990s [10], and typically carry more gene cassettes than mobile integrons, of more diverse functions. In some species the chromosomal integrons constitute a significant fraction of the genome (for example, the total length of the gene cassette pools from five Vibrio chromosomal integrons is equivalent to a small genome) $[1,2]$.

Chromosomal integrons have been found in a wide range of bacterial species and environmental samples. An analysis surveying 603 sequenced bacterial genomes revealed that 9\% carried integrons [7]. Phylogenetic analysis of the integron integrase (IntI) suggests that integrons are ancient structures that have contributed to the evolution of bacterial genomes for hundreds of millions of years, primarily by vertical inheritance $[11,12]$. Horizontal transfer of integrons has also been proposed, as discrepancies are found between the $16 \mathrm{~S}$ rRNA gene tree and the integron integrase tree for the species Vibrio fischeri [13], Shewanella denitrificans, Nitrosococcus mobilis, and the Xanthomonas lineage [7]. At a larger scale, two major clades of integrase were identified, and found to be associated with different environments (soil or ocean), consistent with their exchange among bacterial species occupying common environments $[7,11]$.

Treponema species belong to the spirochete family and many are involved in human diseases: T. pallidum is the cause of syphilis and yaws [14,15]; and the oral pathogen $T$. denticola is associated with periodontal diseases [16,17]. Different from other chromosomal integron-containing species (Vibrio cholerae, Vibrio vulnificus and Vibrio parahaemolyticus) that cause serious infectious diseases, Treponema species are widely found in healthy human populations [5]. By building and examining the phylogenetic distribution of chromosomal integron-containing species among a wider selection of spirochetes, and a phylogenetic tree of integron integrases (IntI) from genomes that contain chromosomal integrons-assisted by an analysis of attC sites-we hypothesize that the chromosomal integron system has undergone at least one gain and one loss in the evolutionary history of the Treponema species. The gain happened after the speciation leading to the two termite-gut-associated species, Treponema azotonutricium and Treponema primitia [18]. The persistent existence of the chromosomal integrons in the human-associated Treponema species, especially in those associated with oral sites, indicate that these species may gain evolutionary advantages by having integron gene cassettes. The loss happened in the common ancestor of T. pallidum and T. phagedenis: the integron system has been entirely deleted in T. pallidum (which has one of the smallest known bacterial genomes), while in the T. phagedenis genome several attC sites are present, but the intI gene seems to have been lost.

\section{Methods}

\section{Building evolutionary tree}

In order to understand the evolutionary history of the Treponema species, we collected the 31 marker genes described in [19] for the Treponema species, as well as for species serving as out-groups. The accession numbers and websites for downloading the draft genomes are listed in Additional file 1: Table S1. The 31 reference genes were extracted from the complete genomes according to their annotations. For unannotated draft genomes, a similarity-based search method (BLAST) was employed to find the genes in the contigs. We translated the genes into amino acid sequences using the standard codon table and aligned them using MUSCLE [20] - separately for each gene. The aligned amino acid sequences were then concatenated and imported into MEGA5 [21] for tree construction, with the model set to JTT (Jones-Taylor-Thornton model, the default amino acid model for MEGA5). The bootstrap number was 500 .

\section{Detecting integrons}

We looked for integron systems by first detecting the presence of the integron intI. To find intI genes, we used genome annotations as well as performing similarity searches using the intI gene sequence from $T$. denticola ATCC 35405 as reference. After collecting intI gene sequences we built a tree for intI from all species that contain integrons, to ask how the integron system has evolved, using the same procedure as for the species tree described above.

Besides looking for intI, we also identified $a t t C$ sites in the genomes, using both similarity-search-based and $a b$ initio approaches. While attI sites are one of the core components of integron systems, they are less conserved than $a t t C$ sites [1], and there is only one attI site in each integron. Thus, we focused on the analysis of attC recombination sites, taking advantage of their conservation and the fact that there are often multiple attC sites in an integron. First, we used eight recombination site sequences that represent all attC sites in the T. denticola ATCC 35405 integron [5] and performed similarity searches with all eight sequences, since not all recombination sites are the same (all attC sites in T. denticola ATCC 35405 genome can be aligned to at least one of the representative sequences with $>85 \%$ sequence identity; the sequences of these eight attC sites are shown in Additional file 1: Table S2). Note that these eight representative $a t t C$ sequences have been used to effectively recover Treponema integron gene cassettes from human metagenomic samples [5]. 
Second, we developed an $a b$ initio approach to identifying chromosomal integrons, by searching for arrays of potential attC sites. Existing computational methods-including the INTEGRALL database [22] and a context-driven approach using a computational grammar [23] — focus on the identification of mobile integrons, especially those of class I, the most widespread and clinically important type. Our method targets chromosomal integrons, utilizing the unique features of the attC sites' secondary structure: a single-stranded att $C$ sequence forms a structure with two stems, an R box and a L box (with one protruding $\mathrm{G}$ ), connected by a loop of varying length [1]. Our method first scans an input genome for regions that are capable of forming the typical attC structure, and then uses these regions' sequences to search for additional potential attC sites (which may be degenerate and thus unable to form the typical attC structure), and finally we keep only the candidates that form clusters (with at least two attC sites within a $10 \mathrm{~K}$ region), to reduce false positives.

\section{Extracting integron gene cassettes}

Except for the annotated T. denticola ATCC 35405 genomes, for which the gene cassettes are described [16], the integron gene cassettes were extracted from the contigs of the draft genomes by identifying the attC recombination sites and extracting genes bounded by these sites. First, the attC recombination sites were detected using similarity searches with the eight representative attC sequences, with identity threshold set to $70 \%$ and coverage set to $50 \%$. We then predicted the cassette genes using FragGeneScan [24], and extracted genes that were exactly bounded by two attC sites (overlap between attC sites and predicted genes was not allowed). We also set the maximum number of genes between any two attC sites to three, which is the maximum number of genes so far reported between any two integron recombination sites [16]. Note that this approach has been applied to human metagenome samples and successfully recovered integron gene cassettes from the metagenomes [5], demonstrating that it is able to effectively collect genes from integron gene cassettes for both complete and fragmented genomic sequences.

\section{Results and Discussion}

\section{Integrons arrived in the Treponema species at least once} in their evolutionary history

We constructed the phylogenetic tree of the Treponema species along with several other spirochete species, as well as several other integron-containing species, as outgroups, as shown in Figure 1. The evolutionary tree suggests that the Treponema species appeared after other selected spirochetes, including Leptospira interrogans, Brachyspira murdochii, Borrelia burgdorferi, Spirochaeta thermophile, and Spirochaeta coccoides. Within the Treponema clade, T. azotonutricium and T. primitia (both termite associated) are the two earliest branching species among sequenced Treponema species.

Integron systems were detected by looking for intI genes in sequenced genomes or contigs (see Additional file 1: Figure S1 for a multiple alignment of predicted integron integrases). Figure 1 shows the phylogenetic tree of the species, with integron-containing species marked with stars, and with paths leading to these species highlighted. From this figure one can clearly see that there is a large gap (more than est. 0.4 MYA) between the two subtrees containing integrons (located at the top and bottom part of the figure): integron systems are missing in all earlier branching spirochetes and only appear in some Treponema species, including all strains of $T$. denticola, $T$. vincentii, $T$. brennaborense, and $T$. succinifaciens. This suggests that the integron system was acquired by the Treponema lineage after $T$. azotonutricium and $T$. primitia-isolated from termite guts-diverged.

We collected intI genes from representative species described in $[7,11]$ and built a phylogenetic tree specifically for the intI gene, as shown in Figure 2. The intI gene sequences of the Treponema species form a unique branch in the tree, indicating that the integron system in Treponema sps. we identified was obtained via LGT only once, and this branch clusters (boxed in Figure 2) with intI genes from Kuenenia stuttgartiensis, Synechococcus sp., Chlorobrium phaeobacteroides, and Blastopirellula marina. These species originate from a variety of environments: Kuennenia stuttgartiensis was isolated from a bioreactor community; Chlorobium phaeobacteroides was isolated from anoxic sulfide-containing water $19.5 \mathrm{~m}$ below the surface of the meromictic Lake Blankvann in Norway [25]; and Synechococcus sp. and Blastopirellula marina were extracted from marine environments; thus the actual transfer path of the integron system to the Treponema species is still unclear, as is the division between soil/freshwater and marine integron types suggested in $[7,11]$.

While typical integrons are inverted [7] with the integrase gene transcribed in the opposite direction to that of the cassette genes (the two transcripts diverge from the central attI site), the Treponema integron loci we have identified share the atypical structure previously characterized in T. denticola [16] with intI transcribed toward the attI (and so in the same direction as the cassette genes), adding evidence to the hypothesis of a single LGT of the integron system into the Treponema species. We note that there are a few transposases identified in the gene cassettes in the T. succinifaciens integron (their insertions into the integron could have interrupted the integron structure), but all the genes 


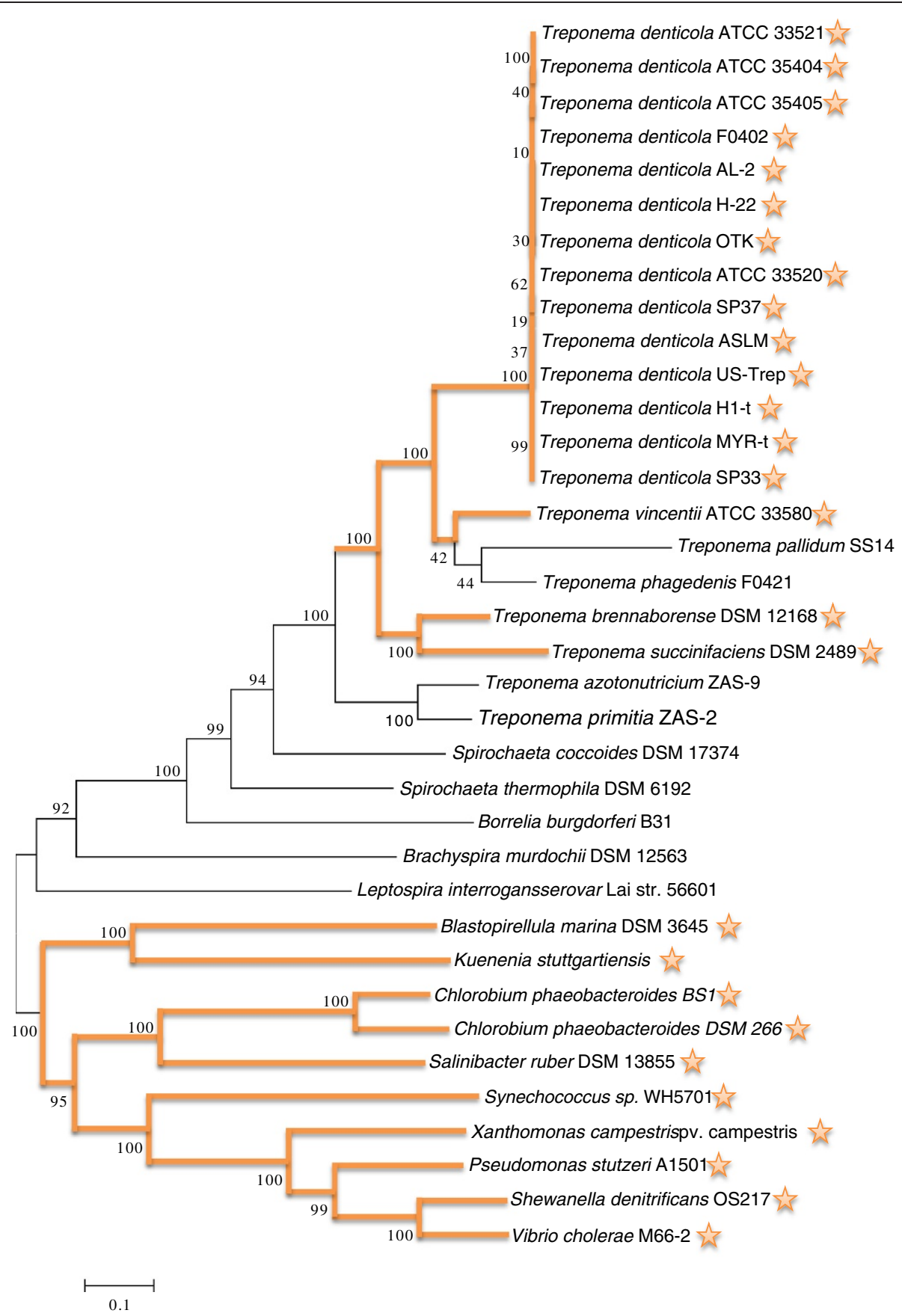

Figure 1 Species tree based on $\mathbf{3 1}$ marker genes. Stars behind species' names indicate that the species carry integrons. Orange lines indicate possible integron evolutionary paths during the divergence of the species.

between the integrase gene (located between 716056 and $717288 \mathrm{bp}$ ) and the closest transposase (located between 736651 and 737865 bp) -with the exception of one gene located between 728835 and $728461 \mathrm{bp}$-are of the same orientation as that of the integrase gene, indicating that the atypical integron structure also applies to T. succinifaciens.
Loss of integron systems in the evolutionary history of Treponema species

Besides the early gain of integrons by the Treponema genus, we also observe the apparent loss of integrons in some Treponema species. The most parsimonious explanation is that-following a single gain into the Treponema lineage-there was a single loss in the 


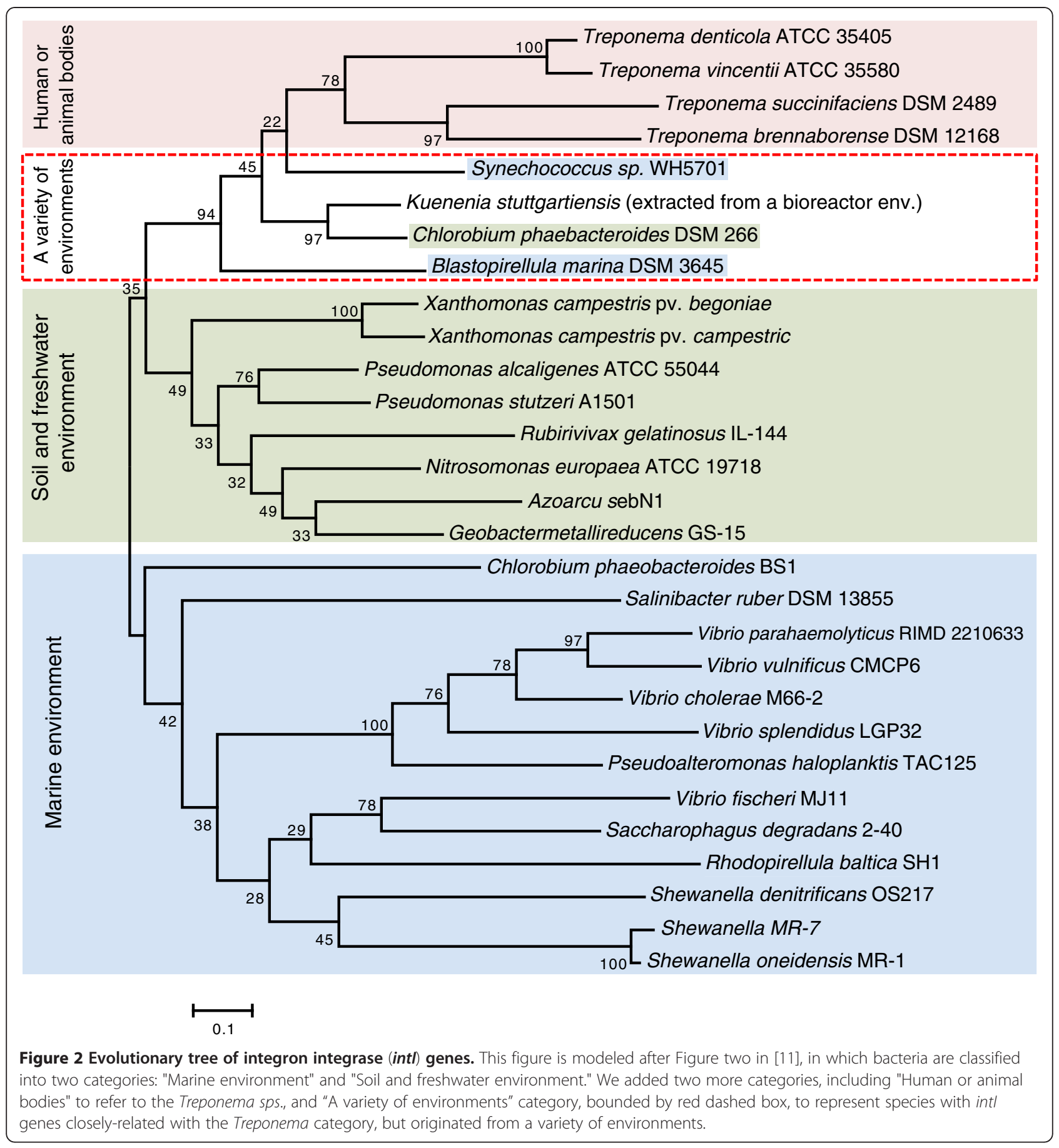

common ancestor of T. pallidum and T. phagedenis. To the best of our knowledge, this loss of an integron system from a lineage is unique-the structure of the Treponema chromosomal integron and its flanking regions are not associated with mobile elements and do not appear to be mobile [1]. Thus our result provides the first instance of the loss of a chromosomal integron in evolutionary history.
Perhaps the most striking result is observed in the genome of $T$. phagedenis. Even though we could not find an intI in this species, we did find integron attC recombination sites at a chromosomal location syntenic to the integron element in other Treponema, and were able to predict integron cassette genes bounded by attC sites (see a comparison between the T. phagedenis genome and the T. denticola genome in Additional file 1: 
Figure S2, which highlights the presence of attC recombination sites and the absence of intI gene in the T. phagedenis genome). The existence of integron recombination sites and gene cassettes indicates that the integron system did exist in the common ancestor of this species and was lost recently in the evolutionary process. We could not find any traces of the integron structure, including intI integrase gene and attC recombination sites, in $T$. pallidum, suggesting that the integron system has been entirely deleted in this species.

\section{Differences of attC recombination sites between Treponema species}

By employing the eight consensus sequences of the $a t t C$ recombination sites to perform similarity searches in the Treponema genomes, we found that all $T$. denticola strains and $T$. vincentii possess these attC sites. However, the same method didn't identify attC sites in the T. succinifaciens and T. brennaborense genomes. After applying an $a b$ initio method to find $a t t C$ sites in the genomes of these two species, we discovered 10 attC sites in T. succinifaciens (from which 3 representative sequences were inferred), and one attC site in T. brennaborense. Figure 3A shows the alignment of predicted attC sites; we focused on the core domains of the $a t t C$ sites for the alignment, considering that the sequence conservation in $a t t C$ sites is restricted to two triplets (AAC and GTT) located in the R" and R' boxes [1]. We only included five $T$. denticola attC representative sequences in the alignment, since the other three lack identifiable AAC sites even though they all share sequence similarity with the attC sites included in the alignment. Figure 3B shows the predicted structures of these identified attC sites: all display the typical stem-loop structure with $\mathrm{R}$ and $\mathrm{L}$ boxes, suggesting that the attC sites found in the $T$. succinifaciens and T. brennaborense genomes are genuine.

Note that we detected only a single $a t t C$ site in the $T$. brennaborense genome (between 1344413 and $1344528 \mathrm{bp})$. However, there is an adjacent intI gene (predicted between 1342819 and 1344015 bp; see Additional file 1: Figure S1 for the multiple alignment of the predicted integron integrases, and the conservation of key residues among these sequences). Between

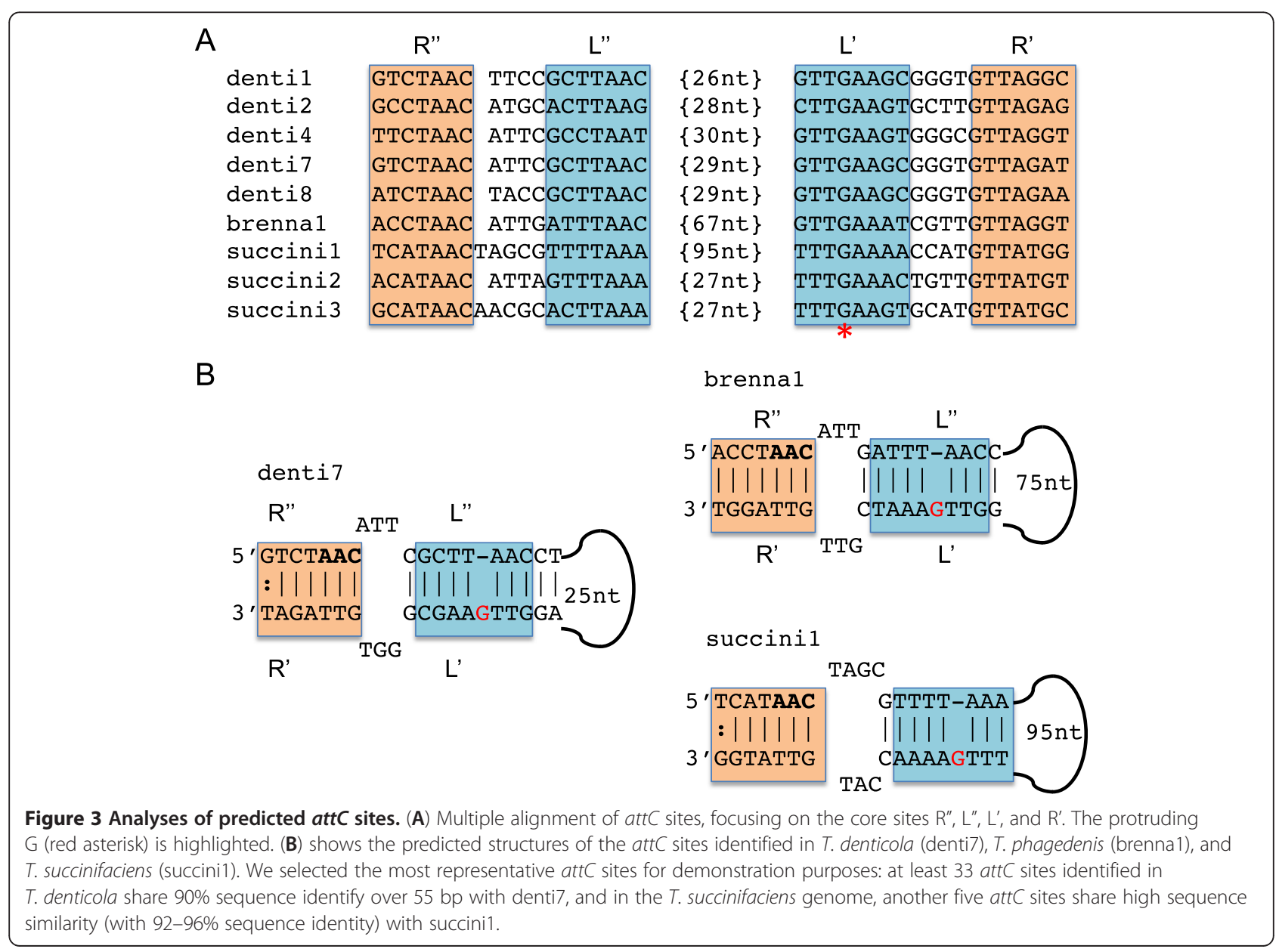


the intI gene and the attC site, we found a small segment (between 1344230 and 1344237 bp) of the same sequence (GTTAGGT) as the R' binding site in the predicted attC site of $T$. brennaborense, consistent with this segment being the $\mathrm{R}$ binding site of the attI site for this integron. All suggest that the integron system in this genome is functional (with integrase gene, attI and attC sites). Chromosomal integron systems lacking integron gene cassettes are found in other species [1], but the lack of a gene cassette array, which is typical of chromosomal integron structures found in other Treponema species, suggests that the integron system in T. brennaborense needs to be examined in additional isolates.

We found that some of the predicted attC sites in T. succinifaciens and T. brennaborense are significantly longer than those in $T$. denticola and $T$. vincentii (in which attC sites are 63-68 bps [1]): the lengths of the three attC site types in T. succinifaciens are $63 \mathrm{bps}$, $68 \mathrm{bps}$, and $132 \mathrm{bps}$ and the attC in T. brennaborense is 115 bps. Although chromosomal integron arraysespecially those with many gene cassettes-typically contain attC sites of similar sizes, arrays have been found that contain attC sites of varying lengths [1]. Note that there are more, long attC sites (6 out of 10) than short ones in the T. succinifaciens genome, and these long attC sites share high sequence similarity (with 92-96\% sequence identity). Since the integron system was inserted into the genome of a common ancestor of the Treponema species (as suggested by Figure 1), and T. succinifaciens and T. brennaborense appeared earlier than T. denticola and T. vincentii (and there are more long attC sites in T. succinifaciens), we hypothesize that the original integron recombination sites were longer (> $100 \mathrm{bps}$ ), and were gradually reduced in T. denticola (63-68 bps).

\section{Dynamics of integron gene cassettes}

With the availability of the $14 \mathrm{~T}$. denticola strains, we are able to compare the gene contents of the integron systems between the different strains (Additional file 1: Table S3 lists attC recombination sites; Additional file 1: Table S4 and Additional file 1: Table S5 list the details and the total number of the integron cassette genes found in these strains). We calculated the proportion of integron genes shared between any two strains by clustering the genes (amino acid identity threshold is set to $70 \%$ ) and show the results as a heat map in Figure 4A. Among the 14 strains, a few are similar to each other in gene content. For example, the three strains MYR-t, ATTC 33520, and H1-t (shown as group III in Figure 4) share more than $60 \%$ of their genes. Other $T$. denticola strains that share large numbers of integron genes include US-Trep and ASLM (group I), and OTK and SP37 (group II). By building a tree based on the 31 marker genes for the 14 strains, as shown in Figure 4B, we found that the sharing of integron genes is largely consistent with the evolutionary relationship among the different $T$. denticola strains: more closely related strains tend to share more genes. For example, strains ATCC 33521 and ATCC 35404, which share $89.7 \%$ of their integron genes, are almost indistinguishable on the phylogenetic tree. Another example is strains US-TREP and ASLM, which share $84.4 \%$ of their integron genes and are closest to each other, as compared to other strains. An exception is that of OTK and SP37, which share $82.6 \%$ of their integron genes (highlighted as group II in Figure 4A) even though they are not close to each other on the phylogenetic tree (see Figure 4B). A possible explanation is that these two species are exposed to similar resources for cassette exchange. Further investigation is needed to understand this phenomenon.

Despite the fact that very closely related $T$. denticola strains share integron genes, the overall integrongene-sharing among all the denticola strains is not very high (on average two denticola strains share $24.79 \%$ of their integron genes; also see Figure 4A). Note that Koenig et al. reported even less sharing of integron genes $(<10 \%)$ among 12 Vibrio isolates [26]. We also reported in a previous work that $T$. denticola integron gene contents are very different between different metagenomic samples [5]. The dynamics of integron genes in $T$. denticola indicate that the integrons of $T$. denticola are fully active and are undergoing active insertion and deletion of cassettes.

Note that for the study of gene cassette dynamics in $T$. denticola (Figure 4A), we only considered genes present between identified attC sites, as attC sites are easier to predict than attI sites (attI sites are less conserved). However, we believe that ignoring the very first gene cassettes between the attI site and the adjacent attC site would not change our conclusions, considering that $T$. denticola isolates contain long arrays of gene cassettes.

\section{Conclusion}

Our combined sequence and phylogenetic analysis of the integron integrases and $a t t C$ recombination sites in Treponema genomes suggest that integron systems have been acquired only once, by the common ancestor of T. brennaborense, T. succinifaciens, $T$. vincentii and $T$. denticola, as we could not find traces of integrons in earlier branching Treponema species (T. azotonutriciums and $T$. primitia, which are associated with termites) or other spirochetes. We cannot exclude the possibility that the integrons have been gained independently in these species; however a single insertion event is more likely 


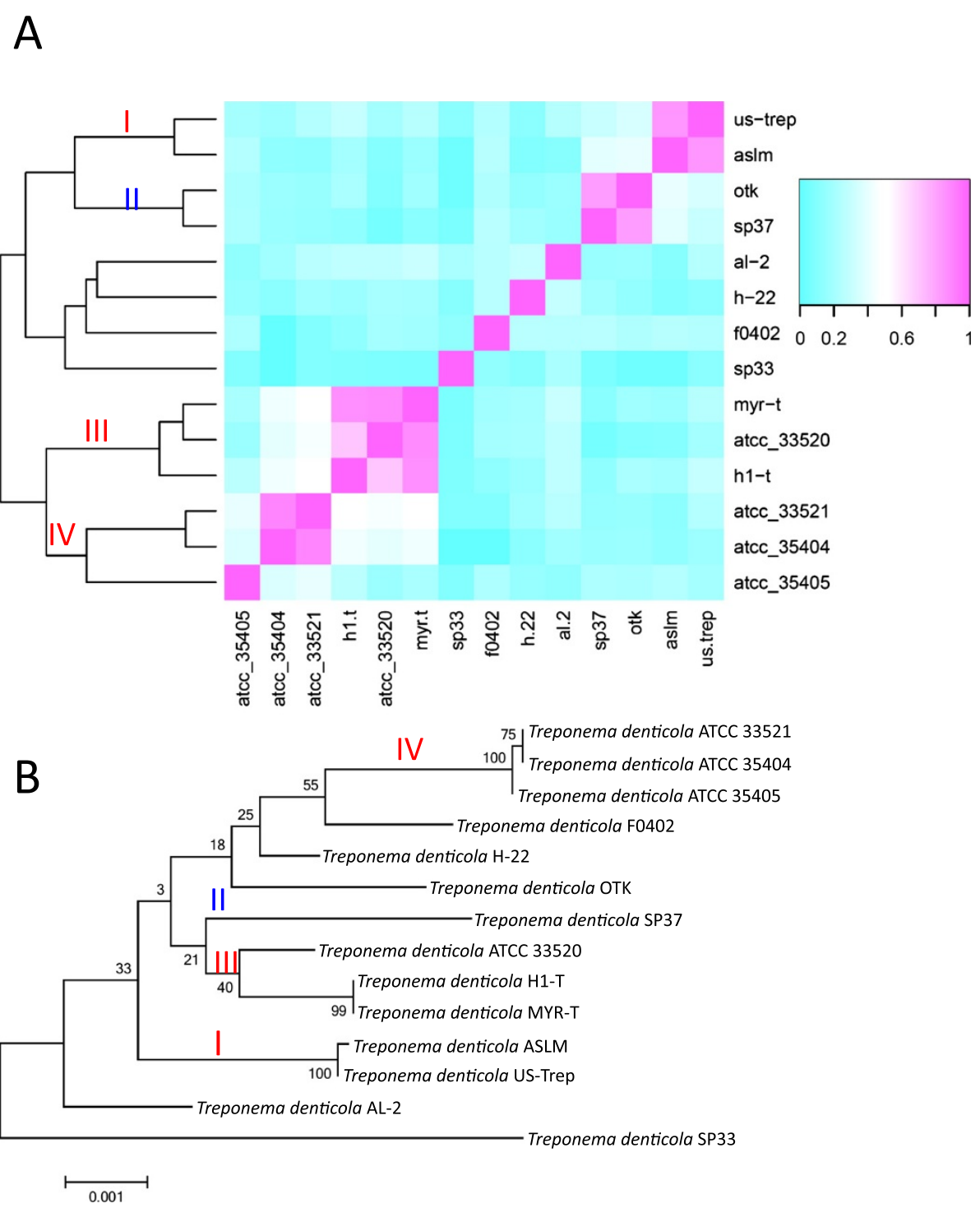

Figure 4 Grouping of $T$. denticola strains based on shared integron gene cassettes and their phylogenetic relationship. (A) Heat map showing the percentage of shared genes between the 14 Treponema denticola strains, with the color spectrum on the right hand side indicating the percentage. The phylogenetic tree shown on the left side of the heat map was generated based on the sharing of the gene cassettes.

(B) The phylogenetic tree of the 14 T. denticola strains, built using the 31 marker genes described in Materials and Methods. Roman numerals in (A) indicate the groups that share significantly more genes as compared to other strains: red numerals indicate that the groupings are consistent with the phylogenetic relationship in (B), and the blue numeral (i.e., group II) highlights an unusual sharing of gene cassettes between not-so-closely-related strains OTK and SP37.

than several distinct events, as the chromosomal integrons are not associated with mobile elements and so cannot move freely, and the Treponema intI genes form a common clade (Figure 2).

We also found evidence for the deletion of integrons in one subclade. The absence of a complete integron structure in $T$. pallidum and $T$. phagedenis can be explained by a single deletion event in the common ancestor of these two species. Moreover, we found remnants of the integron structure, including attC recombination sites and gene cassettes, in the T. phagedenis genome, but not in $T$. pallidum. Without the intI gene this integron structure is now static and cannot facilitate adaptation to new conditions by acquiring new gene cassettes, shuffling of existing gene cassettes, or deletion of cassettes. We could not find traces of an integron structure in T. pallidum, which could be the result of complete degradation of its integron structure.

Our results demonstrate both gain and loss of integron systems in the Treponema species. Even though the horizontal acquisition of integron structures has been suggested by $[7,13]$, to the best of our knowledge no 
literature has provided molecular evidence of integron loss-integrons have been found to be evolutionarily stable. The absence of the intI-along with the integron remnants in T. phagedenis-serves as the first instance of an integron deletion event. Since these chromosomal integrons are not associated with mobile elements, the mechanism of integron insertion/deletion is unknown. With the release of ever more bacterial genomes, we may be able to identify more indel events of integron systems and infer the reasons for these events in the foreseeable future.

\section{Additional file}

Additional file 1: Supplementary materials. This document contains Supplementary Figures S1-S2, and Supplementary Tables S1-S5.

\section{Competing interests}

There are no competing interests.

\section{Authors' contributions}

YW conceived of the study, carried out the analysis and drafted the manuscript. TD participated in the analysis and helped to draft the manuscript. YY conceived of the study, and participated in its design and coordination and helped to draft the manuscript. All authors read and approved the final manuscript.

\section{Acknowledgement}

The genome sequence of T. brennaborense was produced by the US Department of Energy Joint Genome Institute http://www.jgi.doe.gov/ in collaboration with the user community. The authors also thank the Broad Institute for sequencing and releasing the draft genomes of Treponema denticola strains. This work was supported by the National Science Foundation (grant DBI-0845685). We thank the anonymous reviewers for their helpful suggestions.

\section{Author details}

${ }^{1}$ School of Informatics and Computing, Indiana University, Bloomington, IN 47408, USA. ${ }^{2}$ Department of Biology, Indiana University, Bloomington, IN 47405, USA.

Received: 26 July 2012 Accepted: 11 January 2013

Published: 22 January 2013

\section{References}

1. Cambray G, Guerout AM, Mazel D: Integrons. Annu Rev Genet 2010, 44:141-166.

2. Rowe-Magnus DA: Integrase-directed recovery of functional genes from genomic libraries. Nucleic Acids Res 2009, 37(17):e118.

3. Rowe-Magnus DA, Guerout AM, Biskri L, Bouige P, Mazel D: Comparative analysis of superintegrons: engineering extensive genetic diversity in the Vibrionaceae. Genome Res 2003, 13(3):428-442.

4. Stokes HW, Holmes AJ, Nield BS, Holley MP, Nevalainen KM, Mabbutt BC, Gillings MR: Gene cassette PCR: sequence-independent recovery of entire genes from environmental DNA. Appl Environ Microbiol 2001, 67(11):5240-5246.

5. Wu Y-W, Rho M, Doak TG, Ye Y: Oral spirochetes implicated in dental diseases are widespread in normal human subjects and carry extremely diverse integron gene cassettes. Appl Environ Microbiol 2012, 78(15):5288-5296.

6. Labbate M, Boucher Y, Joss MJ, Michael CA, Gillings MR, Stokes HW: Use of chromosomal integron arrays as a phylogenetic typing system for Vibrio cholerae pandemic strains. Microbiology 2007, 153(Pt 5):1488-1498.

7. Boucher Y, Labbate M, Koenig JE, Stokes HW: Integrons: mobilizable platforms that promote genetic diversity in bacteria. Trends Microbiol 2007, 15(7):301-309
8. Martinez E, de la Cruz F: Transposon Tn21 encodes a RecA-independent site-specific integration system. Mol Gen Genet 1988, 211(2):320-325.

9. Hall RM, Collis CM: Antibiotic resistance in gram-negative bacteria: the role of gene cassettes and integrons. Drug Resist Updat 1998, 1(2):109-119.

10. Mazel D, Dychinco B, Webb VA, Davies J: A distinctive class of integron in the Vibrio cholerae genome. Science 1998, 280(5363):605-608.

11. Mazel D: Integrons: agents of bacterial evolution. Nat Rev Microbiol 2006, 4(8):608-620,

12. Rowe-Magnus DA, Guerout AM, Ploncard P, Dychinco B, Davies J, Mazel D: The evolutionary history of chromosomal super-integrons provides an ancestry for multiresistant integrons. Proc Natl Acad Sci USA 2001, 98(2):652-657.

13. Nemergut DR, Martin AP, Schmidt SK: Integron diversity in heavy-metalcontaminated mine tailings and inferences about integron evolution. Appl Environ Microbiol 2004, 70(2):1160-1168.

14. Cejkova D, Zobanikova M, Chen L, Pospisilova P, Strouhal M, Qin X, Mikalova L, Norris SJ, Muzny DM, Gibbs RA, et al: Whole genome sequences of three Treponema pallidum ssp. pertenue strains: yaws and syphilis treponemes differ in less than $\mathbf{0 . 2 \%}$ of the genome sequence. PLoS Negl Trop Dis 2012, 6(1):e1471.

15. Fraser CM, Norris SJ, Weinstock GM, White O, Sutton GG, Dodson R, Gwinn M, Hickey EK, Clayton R, Ketchum KA, et al: Complete genome sequence of Treponema pallidum, the syphilis spirochete. Science 1998, 281(5375):375-388.

16. Coleman N, Tetu S, Wilson N, Holmes A: An unusual integron in Treponema denticola. Microbiology 2004, 150(11):3524-3526.

17. Seshadri R, Myers GS, Tettelin H, Eisen JA, Heidelberg JF, Dodson RJ, Davidsen TM, DeBoy RT, Fouts DE, Haft DH, et al: Comparison of the genome of the oral pathogen Treponema denticola with other spirochete genomes. Proc Natl Acad Sci USA 2004, 101(15):5646-5651.

18. Graber JR, Leadbetter JR, Breznak JA: Description of Treponema azotonutricium sp. nov. and Treponema primitia sp. nov., the first spirochetes isolated from termite guts. Appl Environ Microbiol 2004, 70(3):1315-1320.

19. Ciccarelli FD, Doerks T, von Mering C, Creevey CJ, Snel B, Bork P: Toward automatic reconstruction of a highly resolved tree of life. Science 2006, 311(5765):1283-1287.

20. Edgar RC: MUSCLE: multiple sequence alignment with high accuracy and high throughput. Nucleic Acids Res 2004, 32(5):1792-1797.

21. Tamura K, Peterson D, Peterson N, Stecher G, Nei M, Kumar S: MEGA5: molecular evolutionary genetics analysis using maximum likelihood, evolutionary distance, and maximum parsimony methods. Mol Biol Evol 2011, 28(10):2731-2739.

22. Moura A, Soares M, Pereira C, Leitao N, Henriques I, Correia A: INTEGRALL: a database and search engine for integrons, integrases and gene cassettes. Bioinformatics 2009, 25(8):1096-1098.

23. Tsafnat G, Coiera E, Partridge SR, Schaeffer J, Iredell JR: Context-driven discovery of gene cassettes in mobile integrons using a computational grammar. BMC Bioinformatics 2009, 10:281.

24. Rho $M$, Tang $H$, Ye $Y$ : FragGeneScan: predicting genes in short and errorprone reads. Nucleic Acids Res 2010, 38(20):e191.

25. Pfennig N: Chlorobium phaeobacteroides nov. spec. and C. phaeovibrioides nov. spec., two new species of green sulfur bacteria. Arch Mikrobiol 1968, 63(3):224-226.

26. Koenig JE, Bourne DG, Curtis B, Dlutek M, Stokes HW, Doolittle WF, Boucher $Y$ : Coral-mucus-associated Vibrio integrons in the Great Barrier Reef: genomic hotspots for environmental adaptation. ISME J 2011, 5:962-972.

doi:10.1186/1471-2148-13-16

Cite this article as: Wu et al.: The gain and loss of chromosomal integron systems in the Treponema species. BMC Evolutionary Biology 2013 13:16 